\title{
Brésil 2016, l'écume et les courants profonds
}

\section{Hervé Théry}

OpenEdition

Journals

\section{Electronic version}

URL: http://journals.openedition.org/echogeo/14645

DOI: 10.4000/echogeo.14645

ISSN: 1963-1197

\section{Publisher}

Pôle de recherche pour l'organisation et la diffusion de l'information géographique (CNRS UMR 8586)

\section{Electronic reference}

Hervé Théry, «Brésil 2016, l'écume et les courants profonds », EchoGéo [Online], Sur le Vif, Online since 19 August 2016, connection on 19 April 2019. URL : http://journals.openedition.org/echogeo/14645 ; DOI : 10.4000/echogeo.14645

This text was automatically generated on 19 April 2019.

\section{(c) (i) (9)}

EchoGéo est mis à disposition selon les termes de la licence Creative Commons Attribution - Pas d'Utilisation Commerciale - Pas de Modification 4.0 International 


\title{
Brésil 2016, l'écume et les courants profonds
}

\author{
Hervé Théry
}

1 L'hebdomadaire britannique The Economist avait choisi la crise au Brésil comme thème de sa première couverture de 2016. Sous le titre «La chute du Brésil» et une image de la présidente Dilma Rousseff tête basse, il annonçait une "année désastreuse ». Ce n'était pas sa première couverture consacrée à ce pays et la séquence de celles-ci est révélatrice $\mathrm{du}$ changement de son image dans la presse internationale. Trois d'entre elles représentaient le Christ du Corcovado, qui domine la baie de Rio de Janeiro: sur la première (en novembre 2009) il décollait comme une fusée et le titre était «Le Brésil décolle ». Sur la seconde (en septembre 2013) il retombait en vrille, et le titre était «Le Brésil a-t-il tout gâché ?». Sur la troisième (en avril 2016) la statue levait des deux bras une pancarte marquée « SOS » et le titre était « La trahison du Brésil ».

Bien d'autres journaux ont suivi la même pente et, après avoir porté le pays aux nues, se livrent avec délices au Brazil bashing, le dénigrement sans nuances dont la France a aussi été plusieurs fois la victime. Qu'en est-il en fait, comment peut-on analyser la situation actuelle et relativiser l'idée d'une crise profonde du pays avec quelques clés de lecture plus fondamentales? Sous l'écume de la crise économique et politique actuelle, vers où les courants profonds portent-ils le Brésil ?

\section{Récession, dévalorisation, inflation, crise politique}

Sur le plan économique, 2016 est en effet une mauvaise année pour le Brésil. Selon les estimations publiées le $1^{\text {er }}$ juin 2016 par l'OCDE, après une contraction de 3,8 \% en 2015, son produit intérieur brut (PIB) devrait encore reculer de 4,3\% en 2016 et de 1,7\% en 2017. La projection des principales institutions financières pour l'inflation en 2016 est de $7,06 \%$ et de 5,50 \% pour 2017. La conjoncture économique jusque-là favorable au pays, qui avait largement échappé à la crise de 2008, s'est retournée avec le ralentissement de la demande chinoise, le premier client du Brésil, qui lui vendait d'énormes quantités de minerai de fer et de soja. 
4 Le prix de ces commodities a chuté avec la baisse des commandes passées à la Chine par les pays en crise et l'essoufflement de la demande interne chinoise, ce qui a affecté par rebond les rentrées de devises du Brésil. L'impact a toutefois été atténué par la dévalorisation du Real par rapport au dollar, ses exportations (libellées en dollars) se traduisant par des totaux moins minorés une fois transformés en Reais. De surcroît, la place des exportations dans le PIB brésilien est faible, le marché intérieur en représentant plus de $80 \%$, et c'est donc la stagnation de celui-ci qui est la cause principale de la crise économique, principalement en raison de la hausse du chômage, de l'incertitude politique et des scandales à répétition qui minent la confiance des consommateurs et des entreprises.

5 Lula, le charismatique président des fastes années 2010, avait choisi Dilma Rousseff pour lui succéder et, grâce à l'aura de son mentor, sa popularité était longtemps demeurée forte, jusqu'à la crise de juin 2013 et à la vague de manifestations qui a déferlé dans tout le pays. Passant d'une contestation de l'augmentation des tarifs des transports urbains à celle du coût de la Coupe du monde de football de 2014, puis à des revendications plus générales, comme la lutte contre la corruption, elles avaient témoigné d'un mécontentement de l'opinion, jusque-là peu apparent. Après cette alerte, la fin du premier mandat de Dilma Rousseff avait été difficile, et sa popularité avait continué à s'effriter jusqu'à l'élection présidentielle de 2014, la plus disputée de l'histoire brésilienne, la présidente n'ayant finalement été réélue qu'avec 51,64\% des voix.

6 La première année de son deuxième mandat a été marquée par des scandales dénoncés par l'« opération Karcher » (lava jato) menée par la police fédérale à la demande d'un juge de Curitiba, Sergio Moro. Lancée en mars 2014 elle a mené à l'inculpation de centaines de personnes pour corruption active ou passive, pour un montant total - pour le moment de plus de 40 milliards de Reais (10 milliards d'euros), dont 10 en pots-de-vin (2,5 milliards d'euros). Certains des accusés coopèrent avec les enquêteurs en échange de réductions de peine et ces dénonciations ont rapidement atteint la compagnie pétrolière Petrobras, faisant apparaître de graves collusions entre des directeurs nommés par le Parti des travailleurs (PT) ou ses alliés et les grandes firmes de travaux publics. Des dizaines d'hommes d'affaires - dont les patrons des principales firmes de BTP - ont été mis en prison, ainsi que quelques hommes politiques, dont le trésorier du PT.

7 Il est à noter que ce n'est pas là le premier scandale, il y en a eu d'autres sous les gouvernements antérieurs, y compris celui du mensalão sous Lula. La corruption signale les effets profonds des modes d'organisation politiques du pays, notamment l'impossibilité de gouverner sans former des alliances très larges, souvent contre nature, qui rendent difficile le maintien de coalitions sans versements occultes. Au fond, cette crise révèle plus la maturité d'une société qui n'accepte plus certains comportements que la révélation d'une corruption insoupçonnée auparavant.

8 Avec la révélation de ces scandales et l'aggravation de la conjoncture, la popularité de Dilma Rousseff a continué à baisser : en février 2016 un sondage sur l'action de son gouvernement ne comptait déjà que $5 \%$ d'opinions favorables, contre $79 \%$ d'opinions mauvaises ou très mauvaises. C'est dans ce contexte d'affaiblissement du pouvoir que, le 2 décembre 2015, le président de la Chambre des députés a décidé de donner suite à une demande de mise en accusation de la présidente, pour pedaladas fiscais (manœuvres comptables douteuses, que la loi de responsabilité fiscale assimile à des « crimes ») dans le budget fédéral. Selon beaucoup d'observateurs, c'est le refus du gouvernement de le soutenir qui l'a amené à ouvrir le processus d'impeachment (comme est généralement 
appelée au Brésil le processus de destitution du président de la République). Sa propre crédibilité a été par ailleurs entachée par la découverte de comptes en Suisse, ce qui l'a amené à démissionner en juillet.

9 La procédure d'impeachment a été ouverte en avril par la Chambre des députés avec la majorité des deux tiers requise et le Sénat a donc ouvert le processus de mise en accusation, également à la majorité des deux tiers. La présidente a donc été écartée du pouvoir et son vice-président, Michel Temer, l'a remplacée, à titre provisoire, en attendant le jugement définitif, prévu en août.

Illustration 1 - Maigre manifestation en faveur de Dilma Rousseff le $1^{\text {er }}$ mai 2016 à São Paulo

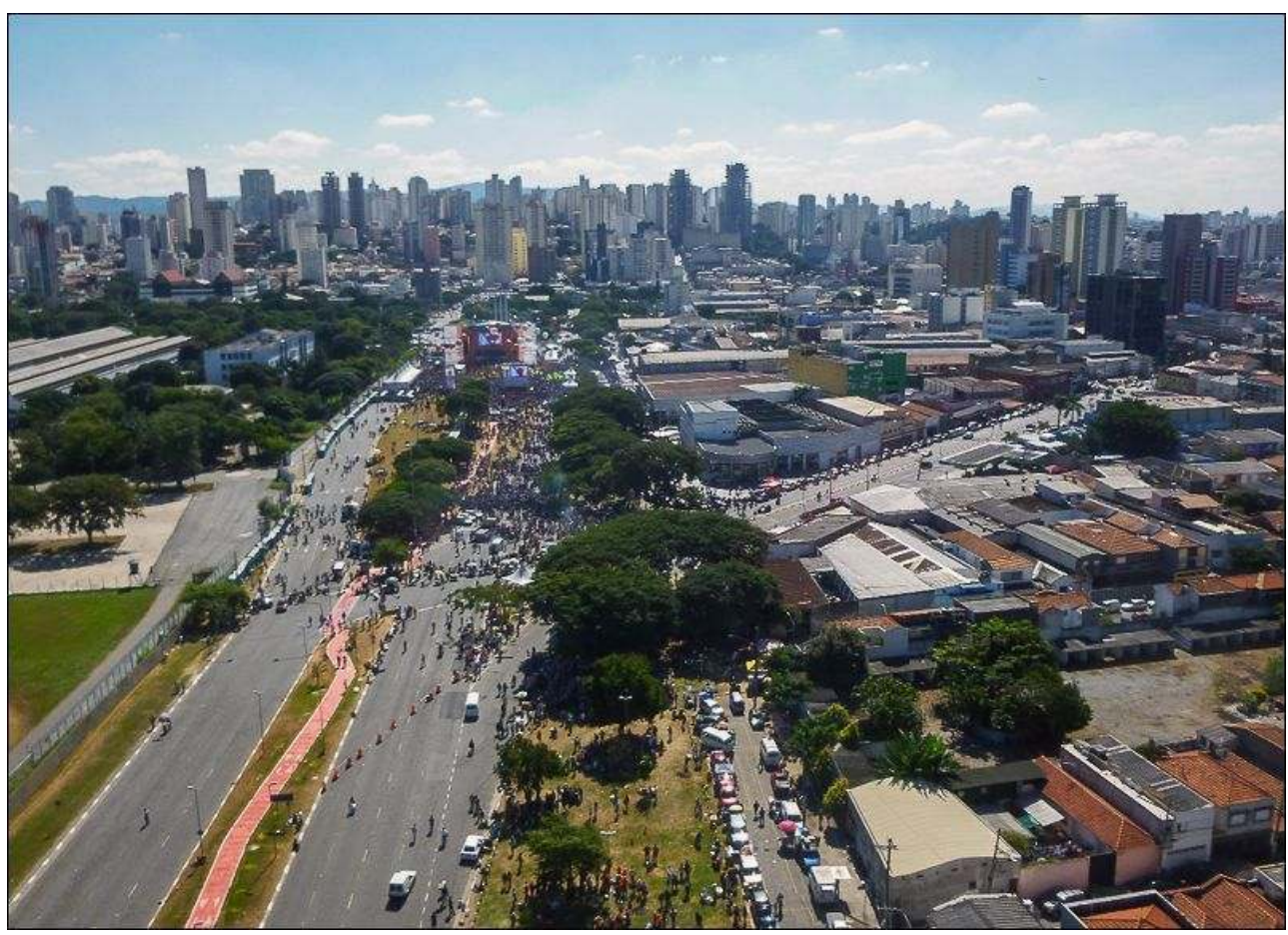

Auteur : Neli A. de Mello-Théry, 2016.

Le ministère formé dans les jours suivants par Michel Temer a été aussitôt critiqué pour être formé exclusivement d'hommes, tous blancs, et pour bon nombre d'entre eux impliqués dans le scandale lava jato. Dans le mois qui a suivi, les mises en cause ont continué et le gouvernement a déjà perdu trois ministres (du Plan, de la Transparence et du Tourisme). D'autres mesures ont été contestées comme la suppression du ministère de la Culture, ensuite rétabli, ou l'ouverture de concours pour le recrutement de 14000 fonctionnaires fédéraux, ensuite annulée.

11 Ce n'est pourtant là que l'écume soulevée par la tempête dans le verre d'eau de Brasília. Pierre Monbeig racontait volontiers que, quand il y vivait dans les années 1930 on disait déjà « Le Brésil grandit la nuit pendant que les politiciens dorment »... Rappelons donc quelques-uns des atouts permanents de ce pays déjà émergé1.

\section{Immensité et ressources agricoles}

Le Brésil dispose d'un des plus grands potentiels mondiaux de terres arables disponibles, de ce point de vue c'est l'un des pays les mieux dotés au monde. Selon des données de 
l'Embrapa et de l'IBGE (équivalents brésiliens de l'INRA et de l'INSEE), sur les 851 millions d'hectares du pays, en tenant compte des surfaces déjà réservées pour la protection de la nature et les réserves indiennes (les superficies occupées par des forêts représentent 449 millions d'hectares, dont 350 millions en Amazonie), 402 millions sont cultivables, et seulement 62 millions sont utilisés par l'agriculture (dont $35 \%$ par des cultures annuelles et $65 \%$ par des cultures permanentes).

Illustration 2 - Camions chargés de soja en attente de déchargement au port de Paranaguá (Paraná)

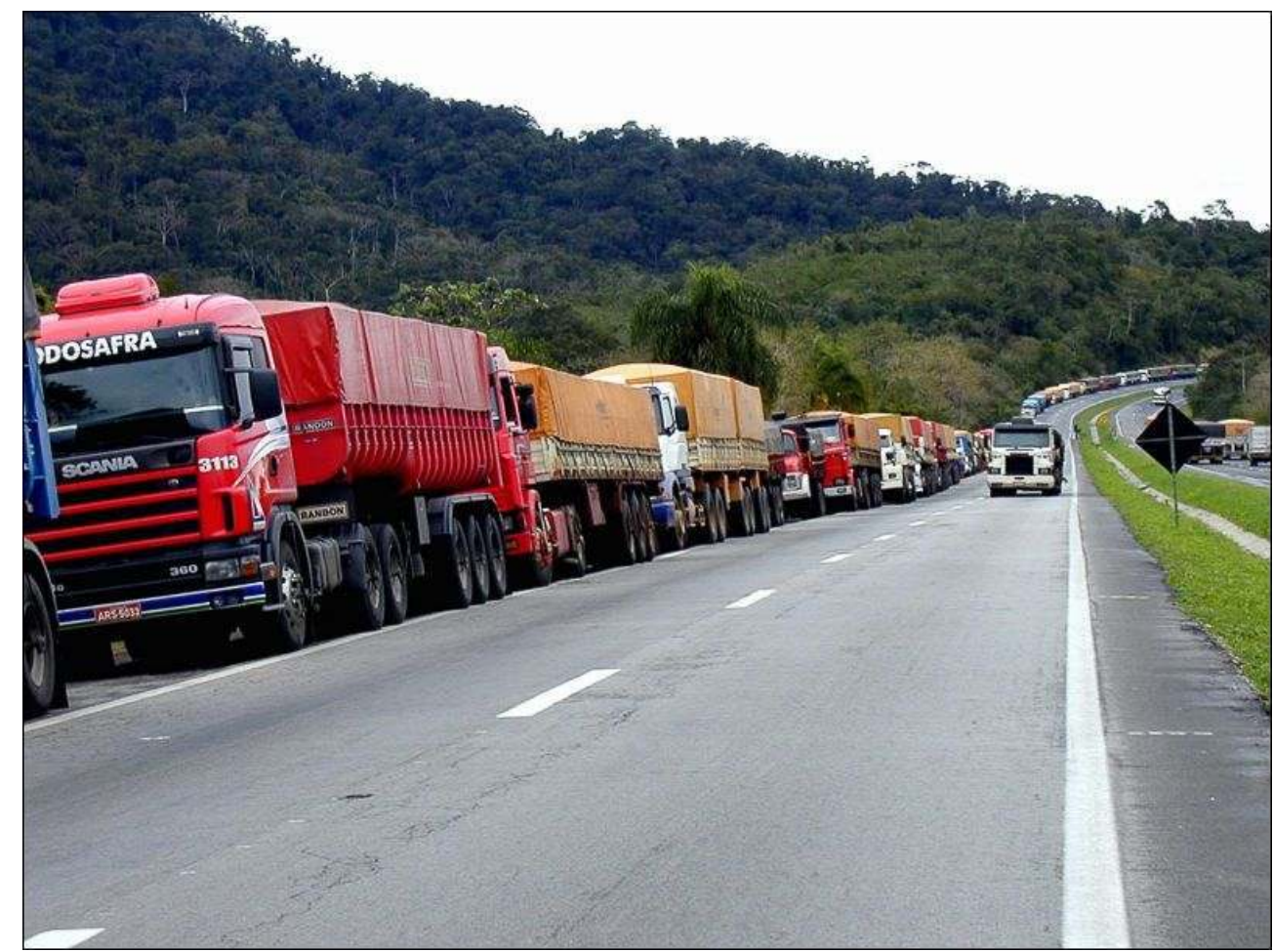

Auteur: Hervé Théry, 2009.

Il resterait donc 340 millions d'hectares à cultiver, dont 90 millions d'hectares immédiatement disponibles (soit trois fois la surface agricole utile française). Ce sont en grande partie des terres non forestières (formations herbacées, savanes, etc.) et il y aurait évidemment des enjeux environnementaux à arbitrer. Ce serait moins le cas pour les terres qui pourraient porter la seconde récolte annuelle qu'autorise le climat tropical (seuls 26 millions d'hectares sur les 62 millions d'hectares cultivés produisent effectivement 2 fois par an) et la récupération des terres dégradées n'aurait que des avantages : on estime que les seuls pâturages dégradés par un usage peu soigneux représentent $600000 \mathrm{~km}^{2}$, soit plus que la superficie totale de la France métropolitaine.

Sans même compter sur ces réserves, le Brésil est déjà très grand pays agricole, car il dispose d'immenses avantages comparatifs : de l'espace, du soleil, de l'eau, de la maind'œuvre d'exécution et d'encadrement, plus les industries d'amont et d'aval qui enserrent l'agriculture dans un puissant complexe agro-industriel. On y produit déjà à peu près toute la gamme des denrées agricoles mondiales, car l'étendue du pays en latitude (38 degrés, de $5^{\circ} 15^{\prime} \mathrm{N}$ à $33^{\circ} 45^{\prime} \mathrm{S}$ ) permet d'y cultiver aussi bien les plantes tropicales que celles du monde tempéré : l'élevage bovin et le palmier à huile apprécient le climat chaud et humide de l'Amazonie; les climats subtropicaux qui occupent le centre du pays 
permettent de jouer à la fois sur les denrées tropicales (canne à sucre, café, arachide) et sur les grains (maïs, soja). Les climats subtropicaux du Sud autorisent les fruits et les légumes de climats tempérés (pomme de terre, blé, avoine, raisin, pommes).

De surcroît il y existe non pas une mais deux agricultures: d'une part une petite agriculture familiale qui a des parentés avec ses homologues européens dans le Sud, ou avec l'Afrique dans le Nordeste, d'autre part un agrobusiness qui n'a rien à envier à ses équivalents nord-américains. Le partage des tâches et des cultures - qui ne va pas sans conflits, souvent violents - entre paysannerie et agrobusiness est clair: la première fournit l'essentiel de l'alimentation du pays, le second les produits d'exportation. La preuve en est que le pays comptait encore récemment deux ministères de l'agriculture, le ministère de l'agriculture, de l'élevage et du ravitaillement (Ministério da Agricultura, Pecuária e Abastecimento), qui s'occupe d'appuyer le puissant secteur agro-industriel, et le ministère du développement agraire (Ministério do Desenvolvimento agrario), chargé d'encadrer la petite agriculture et de promouvoir la réforme agraire. L'actuel gouvernement intérimaire de Michel Temer (en l'attente du jugement de Dilma Rousseff) a intégré le second au "Ministère du développement social et agraire », ce qui indique bien la fonction qu'il remplit. Cette dichotomie met bien en évidence que l'agriculture brésilienne associe des systèmes de production très différents.

\section{La fenêtre démographique}

16 Le Brésil comptait près de 191 millions d'habitants au recensement de 2010, ce qui le mettait, et de loin, au premier rang en Amérique du Sud, devant la Colombie et l'Argentine (respectivement 46 et 40 millions), et au cinquième rang mondial, après l'Indonésie et avant le Pakistan. C'est le résultat d'une progression rapide mais qui a déjà commencé à ralentir : avec un rythme d'accroissement annuel de 1,15\%, le Brésil fait encore partie des pays à croissance relativement rapide, par opposition aux pays d'Europe et à certains de ses voisins, comme l'Argentine et l'Uruguay. L'évolution classique de la transition démographique y est clairement en cours. Jusqu'en 1960, la natalité avait peu baissé, restant stable autour de $45 \%$ depuis le premier recensement, en 1872, alors que la mortalité avait chuté de 30,2 \%o à 13,4 \%o dans les années 1950 : le taux de croissance était alors passé de 1,63\% à 2,99\%. Autour de 1960, la tendance s'est inversée, la mortalité a continué à baisser légèrement (elle est actuellement de 6,7 \%o grâce à la jeunesse de la population plus qu'à ses conditions de vie), mais la natalité a baissé plus encore, tombant à $30,2 \%$ en 1980 .

Comme le ralentissement de la croissance est récent, la structure par âge de la population reste marquée par la prépondérance des jeunes (la moitié des Brésiliens ont moins de 29 ans, contre 39 ans en France), mais le vieillissement de la population est amorcé (cette même médiane était de 19 ans en 1980) et des réformes ont été engagées pour revoir un système des retraites jusque-là assez généreux pour ceux qui avaient la chance d'avoir un emploi régulier. Malgré ce léger vieillissement - à peine engagé - le Brésil a la chance d'avoir devant lui une "fenêtre démographique» bien différente de la situation européenne : la population d'âge actif y sera pour une vingtaine d'années au moins plus nombreuse que l'inactive, tant celles des jeunes (grâce à la réduction de la natalité) que celles des vieux puisque l'âge moyen de la population reste bas. 
Illustration 3 - Foule de jeunes supporters au stade du Morumbi, à São Paulo

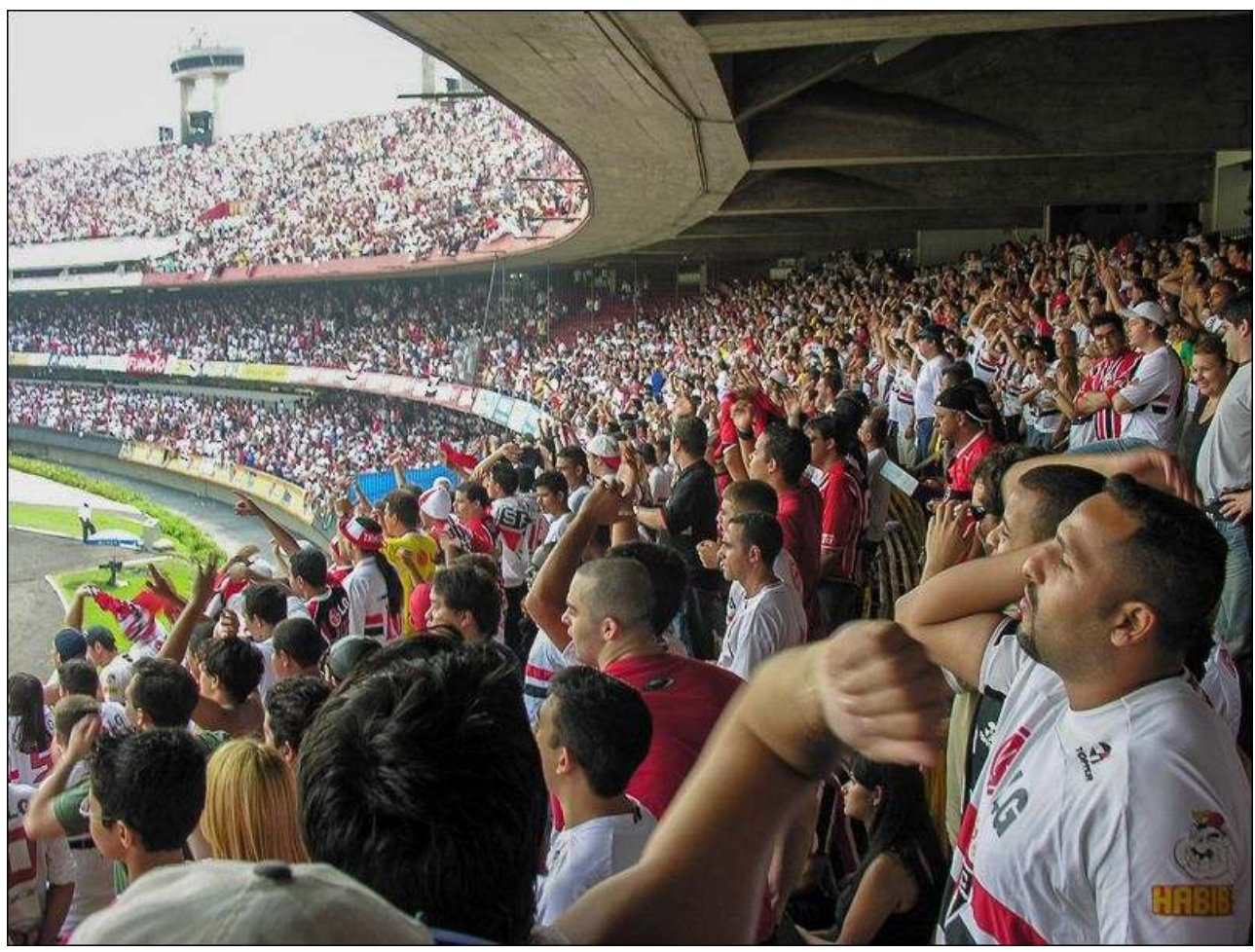

Auteur: Hervé Théry, 2006.

\section{L'indépendance énergétique}

Le Brésil a un avantage décisif dans la compétition mondiale, que beaucoup d'autres pays lui envient: une indépendance énergétique presque totale, grâce au pétrole qu'il a su trouver sur son territoire, à l'hydroélectricité fournie par ses fleuves et aux ressources de la biomasse, principalement l'alcool combustible tiré de la canne à sucre, dont il est le premier producteur mondial. C'est le résultat de deux décisions prises par le régime militaire (1964-1985) lors des chocs pétroliers des années 1970 : chercher de nouveaux gisements sur le territoire national et développer un programme d'alcool combustible. Toutes les deux ont été des succès.

19 Le Brésil produit du pétrole depuis longtemps : découverts en 1939, les gisements de la région du Recôncavo, à proximité de Salvador de Bahia, sont encore en exploitation, mais ils ne produisent plus, avec les gisements découverts ultérieurement dans le Sergipe et l'Alagoas, que $7 \%$ du total national. Sous la pression de la crise pétrolière, le gouvernement militaire avait ouvert une brèche dans le monopole de la Petrobrás, la compagnie nationale créée en 1952 après un grand débat entre nationalistes et partisans de compagnies pétrolières étrangères, signant à partir de 1975 avec ces dernières des « contrats de risque » qui les associaient aux bénéfices en cas de découverte. Elle investit depuis des années près du tiers de son budget dans la prospection, ce qui avait déjà permis des découvertes de gaz et de pétrole au large de Campos (Rio de Janeiro) et de Santos (São Paulo). 

changé la perspective. Grâce à elles, le Brésil n'a plus besoin d'importer de pétrole, tout au plus d'échanger de petites quantités de telle ou telle qualité en fonction des usages recherchés (diesel, essence, kérosène, etc.). Elles ont été rendues possibles par le développement de techniques de forage en eaux profondes qui ont permis à la Petrobras (une des rares compagnies au monde à savoir forer en eaux très profondes) et à ses associés de mieux valoriser les ressources du plateau continental. C'est là, sous 2000 mètres d'eau et 5000 mètres de sédiments, qu'a été découvert en 2007 le gisement dit pré-sal (ainsi nommé parce qu'il est piégé sous une épaisse couche de sel), qui pourrait, s'il tient ses promesses - et tout donne à penser que ce sera le cas - donner au Brésil la sixième réserve mondiale de pétrole et faire du pays - s'il le souhaite - l'un des grands exportateurs mondiaux. Ce pétrole pré-sal ne sera toutefois rentable à exploiter qu'avec un cours assez haut, ce qui n'est pas le cas actuellement. Par ailleurs l'usage des revenus théoriques de ce pétrole ont déjà fait l'objet d'un âpre marchandage sur le meilleur usage à en faire, le mettre en réserve comme l'a fait la Norvège, réserver les royalties à l'éducation, etc., le débat n'est pas clos.

Illustration 4 - Récolte mécanisée de canne à sucre à Olímpia (São Paulo)

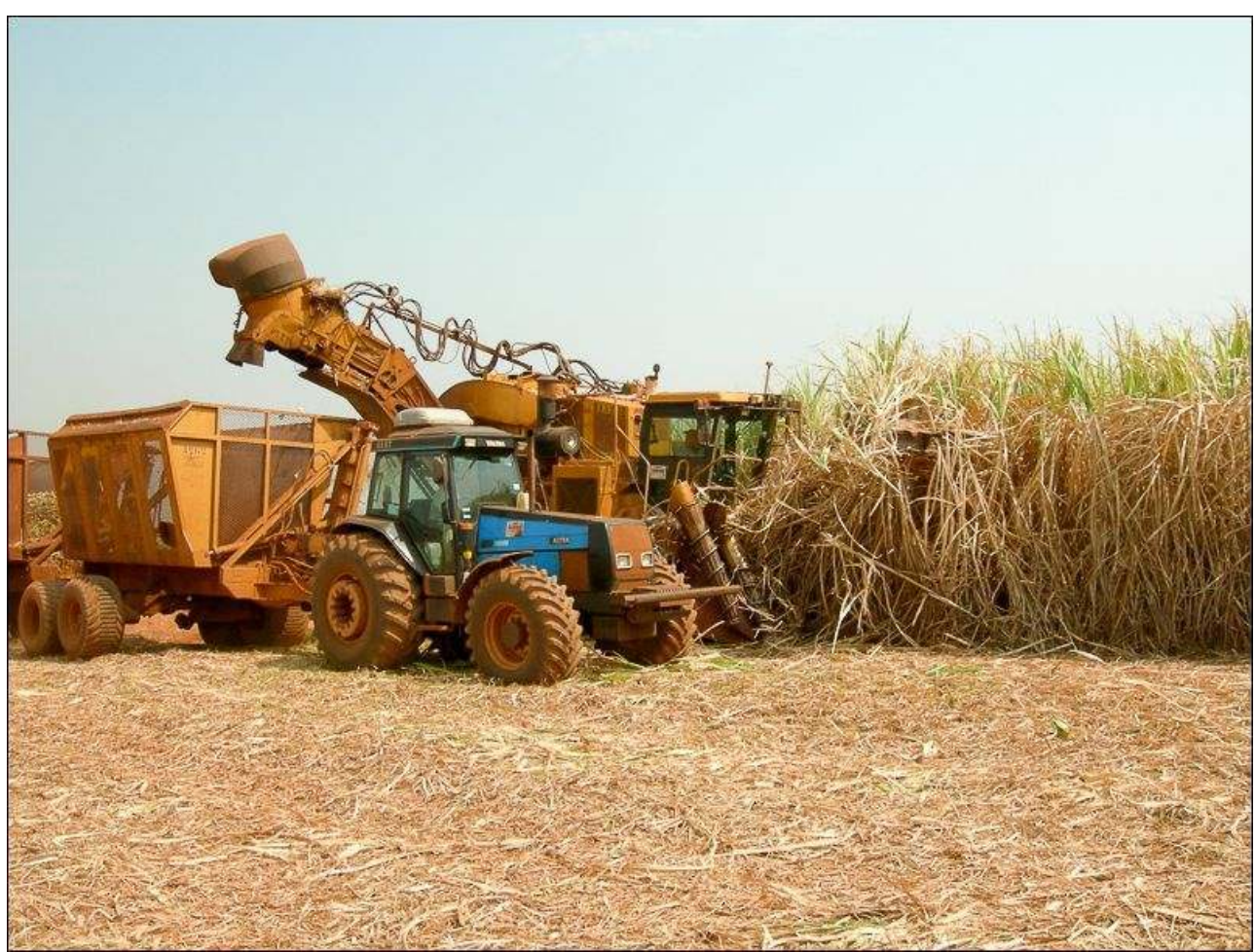

Auteur : Hervé Théry, 2009.

Une des ressources énergétiques principale est toutefois ailleurs, et en rapport avec le gigantisme du pays : pour sa production d'électricité le Brésil dispose d'un formidable potentiel hydroélectrique, qui en assure plus de $70 \%$. Il n'est que partiellement utilisable en l'état actuel des techniques (environ $160000 \mathrm{MW}$ semble-t-il); le Paraná est de loin le fleuve le plus important, son potentiel est colossal, et un effort d'aménagement massif a été consenti en raison de sa situation par rapport aux centres consommateurs. Les compagnies de production électrique du Minas et de São Paulo (la plus importante d'Amérique latine) associées et interconnectées, ont réalisé quelques très grands 
aménagements : le complexe du Rio Grande (3 $400 \mathrm{MW}$ ), celui d'Urubupunga (4 $600 \mathrm{MW}$ ) sont passés au second plan avec la construction du barrage d'Itaipú. En 2015, ce barrage a produit 89,2 TWh, a fourni au Brésil $17 \%$ de l'énergie électrique qu'il a consommée (et au Paraguay près des trois quarts de la sienne). Sa puissance installée (14 $000 \mathrm{MW})$ en fait la deuxième usine hydroélectrique mondiale - le barrage chinois des Trois Gorges a certes un potentiel installé supérieur (18 $200 \mathrm{MW})$ mais sa production est limitée par les irrégularités du débit du Yangtse.

Parmi les énergies nouvelles, c'est l'utilisation de l'alcool de canne à sucre comme combustible qui obtenu le plus net succès. Mélangé à l'essence dans une proportion d'environ $20 \%$, l'alcool réduit la nécessité d'additifs au plomb, ce qui diminue la pollution. Et surtout, on peut désormais l'utiliser seul dans des voitures spécialement modifiées, que tous les constructeurs installés au Brésil savent produire depuis les années 1970. À la suite du plan Proalcool de 1973, plus de 3 millions de voitures équipées de moteurs à alcool avaient circulé dans le pays, et leur proportion à la sortie des chaînes de montage avait atteint jusqu'à $90 \%$, avant de retomber ensuite quand les subventions gouvernementales avaient été réduites, et la production d'alcool avec elles.

Une innovation technique a relancé l'intérêt pour l'alcool, les moteurs dits flex-fuel qui fonctionnent à l'essence, à l'alcool ou à n'importe quelle combinaison des deux. La possibilité d'utiliser indifféremment les deux combustibles a tout changé et les ventes ont décollé dès que les véhicules ont été lancés, en septembre 2003 : dès 2006 elles représentaient $50 \%$ de la flotte en circulation et depuis 2012 près de $90 \%$ des nouvelles voitures vendues au Brésil. Des recherches sont en cours pour utiliser le ricin - une production mieux intégrée à l'économie paysanne, et qui permet l'utilisation de microdistilleries dispersées - et d'autres huiles végétales (soja, arachide, palmier à huile) pour remplacer, au moins en partie, le combustible des moteurs diesel (pour les bus et les camions). Pour le moment elles ne débouchent pas vraiment car les autres usages de ces huiles (alimentaires, industriels) offrent de meilleurs débouchés aux producteurs et le secteur de l'alcool lui-même est en crise avec une diminution du nombre d'usines, en raison notamment de la baisse des cours du pétrole.

À ces avantages massifs et incontestables, et sans vouloir masquer les difficultés que connaît par ailleurs le pays $^{2}$, on pourrait y ajouter des aspects industriels et technologiques (comme le succès de l'Embraer, qui dispute avec Bombardier la place de troisième avionneur mondial, derrière Airbus et Boeing). Ou encore celui d'institutions démocratiques solides, dont la crise actuelle a somme toute confirmé le bon fonctionnement puisque l'impeachment s'est fait dans les règles. Le Tribunal supérieur de justice a en effet rejeté toutes les contestations soulevées par les partisans de Dilma, et plus généralement la justice a pu faire son travail et mettre en cause sans entraves chefs d'entreprises, députés, sénateurs et ministres.

Somme toute, la crise actuelle souligne les lignes de faiblesse de ce modèle de développement - place excessive des exportations, ces dernières années, des minerais et des denrées agricole, corruption de la classe politique -, mais l'on peut espérer que ce sera l'occasion d'y remédier. Dans les premières années de ce siècle, tous les observateurs classaient le Brésil parmi les "pays émergents " promis à un brillant avenir. Sans doute l'analyse était-elle un peu abusive, car il occupe une place singulière parmi eux. Si l'on emprunte - le temps d'une métaphore - le vocabulaire de la géomorphologie, on pourrait dire que pour lui l'émergence ne tient pas de l'isostasie - la lente remontée à un niveau ancien - comme la Chine et l'Inde, qui retrouvent leur rang de grands pays après la 
disparition de la chape de glace qu'a été pour eux la colonisation ou la soumission à l'Occident. Pour lui c'est une vraie surrection, similaire à celle qui soulève progressivement les Andes, sous la pression d'une dérive des continents d'échelle planétaire, une montée lente mais irrésistible et durable.

\section{BIBLIOGRAPHY}

Claval P., 2009. Le Brésil, idées reçues. Le Cavalier Bleu.

Droulers M., 2001. Brésil, une géohistoire. Paris, Presses Universitaires de France.

Endres A., 1999. Histoire du Brésil contemporain : XIXème-XXème siècle. Complexe.

Giblin B., 2016. Les conflits dans le monde, approche géopolitique. Armand Colin.

Le Tourneau F.M., Droulers M., 2011. L'Amazonie brésilienne et le développement durable. Belin.

Théry H., 2016, $2^{\mathrm{e}}$ édition. Le Brésil, pays émergé, Armand Colin.

\section{NOTES}

1. Pour reprendre le titre de mon livre cité en bibliographie.

2. Dans l'ouvrage cité en note 1, elles sont analysées dans un chapitre intitulé e « Le côté obscur de la force »

\section{AUTHOR}

\section{HERVÉ THÉRY}

Hervé Théry, hthery@aol.com, est Directeur de recherche émérite au CNRS, Professeur de posgraduação na Universidade de São Paulo (USP) et co-directeur de la revue Confins (http:// confins.revues.org/). Il publie un blog de recherche Braises (http://braises.hypotheses.org/ ) 